\title{
Perspectivas de las mujeres maltratadas sobre la violencia de pareja en México
}

\author{
Carolina Agoff, Dr en Cs Soc,, ${ }^{(1)}$ Ari Rajsbaum, MC, ${ }^{(2)}$ Cristina Herrera, M en Cs Soc. ${ }^{(3)}$
}

\author{
Agoff C, Rajsbaum A, Herrera C. \\ Perspectivas de las mujeres maltratadas \\ sobre la violencia de pareja en Mexico. \\ Salud Publica Mex 2006;48 supl 2:S307-S3I4.
}

\section{Resumen}

Objetivo. Identificar factores personales, culturales e institucionales que obstaculizan el proceso de búsqueda de salida a la violencia de pareja en mujeres mexicanas. Material y métodos. Entre mayo y noviembre de 2003 se realizaron 26 entrevistas narrativas con mujeres que sufren violencia o que salieron del problema, con perfil de usuarias de servicios públicos de salud en los estados de Coahuila, Quintana Roo y en el Distrito Federal. Resultados. Entre las razones a las que obedece la violencia, según las mujeres, se distinguieron causas (sin voluntad de daño) y motivos (violencia como castigo intencional). Asociados a estas explicaciones se hallan los umbrales de tolerancia y la atribución de responsabilidad de la violencia. Además, el entorno social de las mujeres favorece la reproducción de las normas sociales que pautan la relación entre los géneros y en los que la violencia encuentra su justificación. Conclusiones. Los principales obstáculos para dar solución al problema de la violencia radican en los valores y normas sociales que son compartidos tanto por las víctimas como por el entorno social y los funcionarios públicos.

Palabras clave: mujeres maltratadas; valores sociales; prejuicio; medio social; México

\section{Agoff C, Rajsbaum A, Herrera C.}

Women's perceptions

on intimate partner violence in Mexico.

Salud Publica Mex 2006;48 suppl 2:S307-S3 I4.

\section{Abstract}

Objective. To identify personal, cultural, and institutional factors that hinder the solution to domestic violence. Material and Methods. In Quintana Roo, Coahuila, and Mexico City, 26 in-depth interviews with women currently suffering from intimate partner violence and others who had already found a solution were carried out, between May and November 2003. Results. Among women's explanations to violence, it was possible to distinguish between causes (non intentional violence) and motives (intentional violence). Associated with these explanations, issues related to tolerance emerge, as well as attribution of responsibility. Moreover, the social ties of the women contribute to the acting out of gender roles and the justification or tolerance of conjugal abuse. Conclusions. The dominant values and norms of gender in society, shared by abused women and the community, are responsible for the perpetuation of intimate partner violence.

Key words: battered women; social values; prejudice; social environmental; Mexico

(I) Centro Regional de Investigaciones Multidisciplinarias (CRIM-UNAM). México.

(2) Clínica de Atención a la Violencia Doméstica. Instituto Latinoamericano de Estudios de la Familia. México.

(3) Centro de Investigaciones en Sistemas de Salud, Instituto Nacional de Salud Pública. México.

Fecha de recibido: 26 de agosto de 2004 - Fecha de aprobado: 25 de agosto de 2005 Solicitud de sobretiros: Cristina Herrera. Centro de Investigaciones en Sistemas de Salud, Instituto Nacional de Salud Pública. Av. Universidad 655, Col. Santa María Ahuacatitlán, 62508 Cuernavaca, Morelos, México. Correo electrónico: cherrera@correo.insp.mx 
$\mathrm{L}$ a violencia de pareja* sigue constituyendo un tabú social y cultural, a pesar de ser un fenómeno de gran magnitud en México ${ }^{1}$ y un grave problema de salud pública en el mundo. ${ }^{2}$ Debido a que el ejercicio de la violencia masculina se sigue considerando como algo "normal", 3 con frecuencia resulta "invisible" aun para las propias mujeres maltratadas y esto incide en una inadecuada respuesta al problema. Los elementos que contribuyen a esta negación y naturalización de la violencia son múltiples y de diverso orden: personales, de la relación de pareja, y de orden institucional, social y cultural.

El presente estudio se diseñó específicamente con el objeto de explorar de manera cualitativa algunos de los factores que dificultan una adecuada respuesta al problema de la violencia de pareja. A tal efecto, se llevaron a cabo entrevistas en profundidad con mujeres que sufren violencia por parte de sus parejas, con el propósito fundamental de identificar, a través de sus propias narraciones, los principales factores que actúan como obstáculos, o bien como facilitadores en el proceso de búsqueda de salida al problema de la violencia.

El estudio consistió en la indagación de las perspectivas de las mujeres acerca de cómo entienden la violencia que ejerce su pareja en ellas, qué significados le asignan a este fenómeno, a qué atribuyen el problema y qué orientaciones y estrategias de acción utilizan para su solución. Se trató de recabar información acerca de la comprensión consciente del problema, así como también sobre la denominada "experiencia ateórica"4 o de carácter prerreflexivo, que son conocimientos que orientan la acción y no han sido objeto de una reflexión consciente por parte de los agentes sociales.

El marco teórico dentro del cual se inscribe este estudio, enfoca la problemática de la violencia desde su perspectiva social y no meramente individual o de corte psicopatológico. Se considera que la falta de equidad entre los géneros y la dominación masculina ${ }^{5}$ conforman las raíces del problema de la violencia. Esto se expresa, entre otros aspectos, en valores y normas sociales que orientan el ejercicio de roles de género y que los agentes sociales producen y reproducen en el ámbito de sus vidas cotidianas. La manera en que las afectadas viven y afrontan el problema de la violencia es producto de un proceso social de acción y comunicación, que es responsable de los modos de comprensión del fenómeno en general y de la historia biográfica

\footnotetext{
* En este estudio se han utilizado de manera indistinta los términos violencia conyugal, violencia de pareja y violencia doméstica, para hacer referencia al fenómeno de la violencia ejercida por un hombre contra su mujer, en una relación de convivencia.
}

individual. De esta concepción se deriva que los relatos notificados por las mujeres víctimas de violencia compartan ciertas representaciones del fenómeno como producto del intercambio social del que provienen. Asimismo, se considera que el conocimiento social que orienta las prácticas se distribuye de manera desigual, de acuerdo con la posición que ocupan los individuos en la sociedad, ya sea en términos de su pertenencia de género, clase, étnica, etc. y confiere de manera desigual oportunidades de ejercicio de poder. ${ }^{*, 6}$

\section{Material y métodos}

Entre mayo y noviembre de 2003 se llevaron a cabo entrevistas narrativas a fin de que las mismas mujeres organizaran, a partir de una pregunta estímulo, la narración de su experiencia de violencia de acuerdo con sus propios criterios. El propósito fue realizar un análisis generativo según los principios de la teoría fundamentada, ${ }^{7,8}$ que consiste básicamente en la elaboración de hipótesis emergentes a partir de los datos y en su constatación mediante el estudio comparativo de casos seleccionados en virtud de su valor informativo.

Los investigadores - una socióloga, dos psicólogas y un psicólogo- condujeron y autograbaron las entrevistas con ayuda de una guía abierta de temas, elaborada para explorar algún aspecto particular que no hubiera surgido espontáneamente durante la entrevista narrativa. Los temas de esta guía incluían la historia familiar y de pareja de la mujer -con énfasis en la violencia-; los tipos de violencia que sufre o sufrió; sus percepciones sobre las causas y motivos de la misma; sus ideales de pareja y de género; la trayectoria seguida para buscar salidas al problema de la violencia; sus experiencias con diferentes instancias formales, así como con sus redes informales de apoyo; y los elementos clave de empoderamiento ${ }^{\ddagger}$ que se pudieran observar, especialmente en el caso de aquellas mujeres que fueron capaces de resolver el problema satisfactoriamente.

En el marco del muestreo teórico, ${ }^{7}$ se buscó conformar una muestra ilustrativa de mujeres que hubieran sufrido violencia por parte de su pareja y se encontra-

\footnotetext{
* Se coincide con Hoff, para quien la violencia es un acto social y, en la mayoría de los casos, un comportamiento aprendido en un contexto permeado por inequidades sociales basadas en el género, la edad, la raza, etc.

‡ Este concepto surgió del feminismo para hacer referencia al proceso por el cual las mujeres adquieren capacidades para salir de la subordinación, tomar decisiones que afectan su propia vida y transformar las relaciones de inequidad entre los géneros, pero hoy se aplica por extensión a diversos grupos socialmente vulnerables.
} 
ran ya fuera del problema, y de mujeres que estuvieran sufriendo maltrato al momento del estudio, con el objetivo de identificar -entre otros- los recursos utilizados para darle solución al problema. Asimismo, se buscó que las informantes correspondieran -por su nivel socioeconómico- al perfil de usuarias de los servicios públicos de salud, sin establecer en principio ningún criterio de edad. La muestra abarcó los estados de Quintana Roo, Coahuila y el Distrito Federal, por ser entidades de alta prevalencia de violencia de acuerdo con la Encuesta Nacional sobre Violencia contra las Mujeres 2003 (ENVIM 2003). ${ }^{1}$

El número de casos de la muestra siguió el principio de saturación teórica, 7 para lo cual se contempló, entre otras cosas, la búsqueda de casos negativos. Se llevaron a cabo un total de 26 entrevistas con mujeres entre 23 y 72 años en las tres entidades señaladas.

Se estableció contacto con las informantes en los servicios públicos de salud, con ayuda de las trabajadoras sociales y a través de instituciones civiles, privadas y públicas (refugios) que se dedican a atender a mujeres víctimas de violencia. Estas últimas se encontraban bajo tratamiento. A las que nunca habían recibido atención por el problema de la violencia, se les proporcionó un directorio con instituciones de atención. En todos los casos se obtuvo un consentimiento informado y se trabajó en condiciones de privacidad.

\section{Resultados}

El análisis de los resultados puso en evidencia que la violencia del hombre contra la mujer en una relación de convivencia es un problema que involucra, además del vínculo de pareja, un conjunto complejo de relaciones con el entorno social que favorecen la aparición de la violencia y contribuyen a perpetuarla. La violencia de pareja resulta la manifestación más radical de la inequidad de género y dominación masculina y se rige o comporta según determinadas orientaciones, valores y normas, que establecen derechos desiguales para el hombre y la mujer. Esto se manifiesta no sólo al interior de la relación de pareja, sino también a través de los agentes sociales del entorno, quienes producen y reproducen cotidianamente estas concepciones de género. A pesar de lo anterior, y debido a la precariedad emocional de la mujer originada en el maltrato, es el mismo entorno y los recursos que éste puede eventualmente facilitar lo que constituye una clave para la salida a este problema.

En el análisis que a continuación se presenta, se analizan dos aspectos en particular: a) la percepción subjetiva de las mujeres sobre las razones de la violencia experimentada y b) el problema de los obstáculos para superar la violencia. En relación con este último aspecto, resultó interesante observar la presencia de redes tanto informales como formales que colaboran $u$ obstaculizan la trayectoria de búsqueda de ayuda.*

\section{Las razones percibidas de la violencia}

La percepción subjetiva de la mujer acerca de las razones a las que obedece la violencia que ejerce su pareja contra su persona está directamente asociada al modo en que ella vive este maltrato, así como también a los umbrales de tolerancia experimentada hacia la violencia. ${ }^{\ddagger}, 9$ Asimismo, el análisis de las explicaciones subjetivas que brindan las mujeres entrevistadas resulta crucial para entender las acciones que se llevan a cabo en la búsqueda de una solución a la violencia masculina.

El análisis de las entrevistas permitió diferenciar, como parte de las razones de la violencia percibidas por las mujeres, entre motivos y causas. Así, se puso en evidencia que los motivos involucran intenciones conscientes y voluntad de maltrato por parte de la pareja masculina, mientras que las causas responden a fuerzas externas, ajenas a la voluntad del hombre violento.

Entre las causas de la violencia se mencionan el machismo reinante en la sociedad, pero también problemas no resueltos en la infancia del cónyuge o conflictos con la familia de origen, presiones laborales, características psicológicas como la inseguridad o la necesidad de ser reconocido, y el consumo de alcohol. En sus relatos, estas causas asociadas al maltrato parecen desresponsabilizar en parte a la pareja, quien supuestamente la maltrata impulsado por cuestiones que van más allá de él:

Cuando se molestaba o discutía con sus hermanas, se venía y se desquitaba conmigo (mujer de 26 años del Distrito Federal).

Hay mucho machismo, sí, la mentalidad de los hombres es de que todavía las mujeres como que no valen, no valemos lo mismo que ellos (mujer de 26 años del Distrito Federal).

Era mucha la presión. Cada cuatro días tenía que entrar a trabajar a la noche. También el no dormir bien,

* En la ENVIM se publicó un panorama del total de los hallazgos del estudio.

₹ El ámbito de las razones o justificación de la violencia establece el umbral de tolerancia aceptado. Un estudio sobre violencia doméstica en Chennai, India, demuestra que los umbrales de aceptación de la violencia están dados por el tipo de intensidad, de justificación y frecuencia del maltrato. 
el no descansar, las broncas económicas, todo se le fue juntando y estalló conmigo (mujer de 27 años de Saltillo).

Este tipo de explicaciones, que ubican el origen de la violencia en factores ajenos a la relación de pareja, sugieren dos hipótesis que merecen ser contrastadas en otros estudios. Por un lado, la atribución de responsabilidad del maltrato a la pareja es parcial: las mujeres encuentran una justificación al maltrato de sus parejas, quienes las agreden sin intención, sino más bien movidos por causas ajenas a su voluntad y como expresión incontrolada de afectos. ${ }^{* 10}$ Por otra parte y dado que no es la mujer quien "despierta" las manifestaciones de violencia, su autoimagen no resulta tan comprometida.

Es posible observar que cuando se ubica el origen de la violencia masculina en fuerzas externas al hombre, las mujeres muestran un mayor grado de tolerancia a la violencia de que resultan objeto. Además, el análisis comparativo por edad de las mujeres entrevistadas evidencia que aquellas menores de 35 años se muestran más tolerantes que las mayores, lo que parece sugerir que la mayor cantidad de años de maltrato no favorece la justificación del maltrato por estas mismas causas.

Por el contrario, los motivos revelan intenciones de violencia con fines diversos: el maltrato pareciera funcionar como un mecanismo disciplinar, cuyo objetivo es encauzar, imponer o castigar ciertos comportamientos de la mujer y encuentra su justificación en las normas sociales que pautan el desempeño del rol femenino dentro de la pareja.

\section{La violencia como castigo merecido}

El análisis de las entrevistas permitió observar que las normas sociales que gobiernan la relación conyugal se hacen explícitas a través de los argumentos del conflicto y éstas se expresan, en términos generales, como un ejercicio irrestricto de la voluntad del hombre y un control sobre el comportamiento de la mujer. Asimismo, estas normas establecen la distribución de responsabilidades y obligaciones de cada uno de los cónyuges y ponen en evidencia la relación de profunda inequidad existente entre la mujer y el hombre.

En este contexto se ubican los motivos de la violencia masculina como intención consciente de castigo

\footnotetext{
* Otras investigaciones han constatado la creencia, en hombres y mujeres, de que la violencia no intencional es aceptable y que sin intención no hay atribución de responsabilidad.
}

del hombre por el incumplimiento de las expectativas de rol de género femenino. ${ }^{*, 11}$

Algunas mujeres se sienten en falta y aquejadas de sentimientos de culpa cuando el hombre justifica su maltrato por el incumplimiento de sus obligaciones domésticas. Dado que parece incuestionable que el ámbito doméstico es exclusiva responsabilidad de la mujer, las expectativas de comportamiento femenino no cumplidas convierten la violencia en un castigo "merecido":

Me echa la culpa a mí: "es que tú no atiendes a los niños, es que tú no limpias la casa, es que la comida se echa a perder, es que no se qué". Le digo: ¿es que sabes qué? tú me das veinte pesos para comer, todo el día veinte pesos (mujer de 38 años de Saltillo).

Además, el sentimiento de desconcierto vinculado a la violencia como castigo inmerecido, hace evidente que la relación de pareja establece las pautas de lo que sí puede ser castigado, en caso de incumplimiento. La vecina de una de nuestras entrevistadas se solidariza con la mujer golpeada y dice lo siguiente al golpeador:

La trae como su sirvienta y no como su esposa, y ahora usted le pegó muy feo sin que se lo mereciera. Ella no anda haciendo nada malo (mujer de 44 años del Distrito Federal).

El control que el hombre ejerce sobre el comportamiento de su mujer también se hace evidente fuera del ámbito doméstico y se observa en la imposición y reglamentación de los modos de vestir de la mujer, en la prohibición de relacionarse con vecinas o amigas (menos aún con personas del otro sexo), y en la prohibición de trabajar:

Le dije: ¿sabes qué?, me voy a ir a trabajar. "Ah no dice- tú no te vas a trabajar, yo ya me quiero casar contigo". Y no me dejó, no me dejó (mujer de 72 años del Distrito Federal).

Mantenerse fiel al marido es otra obligación de la mujer, pautada por los valores y normas sociales que orientan los modos de relación entre los cónyuges. La

\footnotetext{
* Las representaciones sociales acerca de los roles de género expresados en el deber ser de las mujeres en sus relaciones de pareja, así como la exploración de las circunstancias donde es justificable hacer uso de la violencia desde la perspectiva de las propias mujeres, muestran los niveles de tolerancia social que encubren la identificación de la violencia y contribuyen a su perpetuación dentro del ámbito privado.
} 
violencia masculina originada en la sospecha de infidelidad ocupa un lugar central entre los motivos percibidos. Algunas mujeres suelen interpretar este tipo de violencia como expresión de celos de parte de su pareja. Sin embargo, una mirada más atenta permite advertir que el tema de la infidelidad no debe situarse necesariamente en el ámbito de los sentimientos de ambos cónyuges, sino en el de los intereses que la unión conyugal representa para cada uno: se trata de que ella no transgreda una norma que lo colocaría a él en una situación de poca hombría y de dignidad manchada. En el caso de la mujer, la amenaza de que él le sea infiel conlleva el riesgo de perder eventualmente la manutención para ella y sus hijos y/o el estatus social que le confiere tener una pareja estable y unida. Con frecuencia, se observa la firme creencia de que una pareja es exitosa mientras permanezca unida, independientemente de la existencia de violencia. El riesgo de que la infidelidad del hombre se transforme en abandono, sin embargo, favorece durante largos períodos la tolerancia a la violencia.

La obligación marital de la mujer de mantener relaciones sexuales con su pareja, e independientemente de su propio deseo, también lleva, con frecuencia, a la violencia sexual:

Me quiso forzar que tuviera relaciones sexuales con él. Yo le dije que no quería y él me dijo que era mi obligación, que por eso yo me había casado (mujer de 34 años de Chetumal).

Tenía relaciones sexuales a fuerza, porque si yo no accedía, era motivo de que me pegaba o también decía: "no quieres acostarte conmigo porque, este, sabe quién con quién te estás acostando. Eres una cualquiera" (mujer de 38 años de Saltillo).

Así, la violencia masculina de tipo físico, emocional, sexual y económico, sigue el patrón de reencauzar la conducta femenina y reestablecer tanto las reglas del poder que el hombre detenta, como la sumisión de la mujer. Este tipo de explicaciones subjetivas permiten observar una clara atribución de responsabilidad del maltrato al hombre abusador, como también un umbral de tolerancia a la violencia menor. Sin embargo, no resulta evidente que estos dos aspectos favorezcan una salida precoz al problema de la violencia; los obstáculos para solucionarlo son, sin duda, múltiples. A continuación se analizarán aquellos que provienen del entorno social de las mujeres.

\section{La trayectoria de búsqueda de ayuda}

En el ámbito de investigación de la violencia contra las mujeres, se ha resaltado que el aislamiento social constituye un factor de riesgo considerable vinculado a la violencia de género. ${ }^{2,12} \mathrm{El}$ aislamiento puede favorecer la aparición de la violencia, como también puede contribuir a perpetuarla en el tiempo, cuando la mujer se ve obligada por la misma dinámica de la violencia conyugal a dejar de lado sus relaciones. Esto obedece posiblemente a que la dinámica de relación de la pareja está menos abierta al escrutinio de las personas del entorno. $^{13,14}$

Asimismo, se hace evidente que, debido a la precariedad emocional que caracteriza a las mujeres violentadas, ${ }^{*, 15}$ resulta de fundamental importancia contar con la presencia de redes de apoyo que faciliten la ayuda necesaria para salir de este círculo de violencia. En general, las mujeres toleran diferentes formas de violencia durante mucho tiempo y sólo con el transcurso de los años y con ayuda de otros, aprenden a visualizar el maltrato y cuestionan ese modelo aprendido del "hombre que manda":

\begin{abstract}
...ahora sí estoy aprendiendo, estoy oyendo. Porque ahora sí yo vengo por decir de un pueblo, pues ahora sí me inculcaron, el hombre lo puede hacer y lo puede deshacer y uno de mujer pues tiene que, pues ahora sí, que todo lo que diga el hombre que tiene que hacer uno. Yo cuando vine aquí pues ahora sí mi esposo desde que me trajo acá, me estoy dando cuenta que él sí me trató muy mal (mujer de 44 años del Distrito Federal).
\end{abstract}

Empero, cabe señalar que la sola presencia de redes sociales no siempre resulta una fuente de apoyo. ${ }^{\ddagger}, 16$ Los datos empíricos revelan que no sólo debe atenderse al tema de la ausencia o presencia de las redes sociales en relación al fenómeno de la violencia de pareja, sino a la naturaleza y calidad de estos vínculos. En el ámbito de discusión sobre capital social, es decir, de "aquellos recursos reales o potenciales que provienen de la participación en redes de relaciones más o menos institucionalizadas de conocimiento y reconocimiento mutuo", 17,18 se ha discutido críticamente en torno a los beneficios que éstas aportan, así como también los perjuicios que, como en este caso, se evidencian bajo la forma de un control social que refuerza los roles tradicionales de género. Aquí interesa, en particular, mostrar el tipo de reacción y argumentos que ofrecen las personas del entorno de la mujer ante el fenómeno de la violencia,

* Las mujeres maltratadas suelen presentar síntomas de un desorden de ansiedad llamado "desorden de estrés postraumático". Las alteraciones causadas por este desorden pueden resultar en incapacidad para el funcionamiento social y laboral.

$\ddagger$ El concepto de apoyo social ha sido usado ampliamente, aunque aún no está claramente delineado (referencia 16). 
para dejar en evidencia los aspectos negativos de cierto tipo de vínculos.

\section{Las redes sociales informales}

Las mujeres víctimas de violencia suelen aislarse cada vez más del entorno social, especialmente para evitar explosiones de violencia causadas por los "celos" de la pareja. Con ello pierden mucho de su capital social y se encierran aún más en el círculo de la violencia:

Me decía malas palabras, de las peores palabras, pues ya últimamente ya he dejado todo eso con tal de no tener problemas, o sea que ya no salgo para que no me hablen (mujer de 32 años de Chetumal).

Algunas personas del entorno, según las entrevistadas, parecen colaborar en un proceso de salida cuando intervienen para "abrirles los ojos", como expresan ellas mismas en sus relatos. ${ }^{*}, 19$ En este caso, las redes sociales funcionan como capital social positivo, ayudando a la mujer a evitar la violencia, a salir de ella o a no reincidir si ya están en proceso de salida del problema.

No obstante, el análisis de las entrevistas puso en evidencia, con marcada frecuencia, la presencia de "capital social negativo": ${ }^{18}$ se trata de aquellas relaciones personales muy significativas para la mujer (como sus padres, hermanos o amistades) cuyos "consejos" la orientan a soportar la violencia como algo natural o como un destino, o bien aquellos que abiertamente se colocan del lado del agresor. En estos casos, el entorno social reproduce y refuerza las normas tradicionales de género. En particular la familia juega un papel fundamental en la reproducción de la ideología de género a través de las expectativas y sanciones sobre el cumplimiento de roles prescritos: ${ }^{\ddagger}$

No, mire, antes yo no le platicaba a la gente la situación que estaba pasando, pero la mayoría de la gente se daba cuenta, incluso mi madre, ¿sabe? Se daba cuenta pero ella decía que ese era el papel de la mujer, que nosotros teníamos que obedecer a nuestro esposo. Y yo le decía: es que no puede ser, ¿cómo voy a aceptar a alguien que me golpea? ¿Cómo voy a vivir así? (mujer de 40 años de Saltillo).

\footnotetext{
* Se ha documentado la importancia del apoyo social para una salida exitosa al problema del abuso, entre otros, porque posibilita el autorreconocimiento de la mujer como mujer maltratada.

$\ddagger$ Agoff C, Herrera C, Castro R. Reversing Granovetter theorem: the weakness of strong ties and its perpetuating effects on gender violence. Violence Against Women (sometido a dictamen).
}

Es que José me pega muy feo, mamá, yo no quiero vivir con él. Dice: "pues es tu cruz hija, te tienes que aguan$\operatorname{tar}^{\prime \prime}$. Entonces al escuchar eso dije, yo qué gano con irle a decir... Entonces por eso siempre me quedé callada de todo lo que mi pareja me hacía. O sea, yo solita, pues, me guardaba todo (mujer de 43 años del Distrito Federal).

Los valores y normas sociales que establecen las pautas de comportamiento femenino, y que se reproducen y transmiten en el ámbito familiar (entre otros), imponen a la mujer un imperativo de sometimiento a la violencia que se expresa de dos modos: como justificación de la violencia como castigo merecido por el incumplimiento del rol de género prescripto y/o como tolerancia hacia el maltrato por aceptación de un destino natural de toda mujer.

Por otra parte, la pobreza de capital social se hace muy evidente en los casos de las entrevistadas que han migrado y no cuentan con redes sociales de ningún tipo:

Yo me vine porque tuve la oportunidad de poder escapar. Me escapé como quien dice porque él nos tenía como secuestrados, porque yo de mi familia no sé nada. Yo tengo años que no veo a mi familia (mujer de 30 años de Saltillo).

La migración suele ser un factor relacionado con el agravamiento de la violencia, precisamente por la pérdida de redes que este movimiento implica. Se ha observado que las mujeres que no poseen redes sociales de ninguna naturaleza recurren a instituciones formales en busca de ayuda, con mayor frecuencia que aquellas mujeres que cuentan con redes, aunque éstas no constituyan una fuente de apoyo.

\section{La búsqueda de ayuda institucional}

La trayectoria de búsqueda de ayuda institucional es parte de lo que se conoce como "ruta crítica", ${ }^{20}$ es decir, el propio proceso de conscientización y búsqueda por parte de la mujer, y los recursos sociales e institucionales con los que pudo contar o no, para dar solución a su problema. ${ }^{*, \neq}$

\footnotetext{
* El análisis de las llamadas rutas críticas pretende identificar los factores que alientan o desalientan la denuncia, para lo cual se contemplan aspectos personales y del entorno (estos últimos comprenden desde el apoyo de familiares, hasta las campañas en los medios de comunicación y el trato y apoyo institucional).

$\ddagger$ COVAC. Asociación Mexicana contra la violencia a las mujeres A.C. Estudio sistémico de la ruta crítica de atención a mujeres afectadas por violencia. 22/03/ 2002. Documento no publicado.
} 
Las mujeres entrevistadas han informado no acudir a los servicios de salud para solicitar ayuda. Suelen evitar hacerse "visibles" en los servicios de atención por vergüenza o desconfianza, y cuando acuden por lesiones o malestares causados por la violencia, constatan que los médicos se muestran indiferentes. En contraste, las psicólogas o las trabajadoras sociales que laboran en estos mismos servicios les merecen más confianza, en especial porque muestran un mayor interés o voluntad de ayudar:

El médico pues, no tanto que no confíe, sino que el médico solamente nos revisa, ¿verdad? Porque me ha tocado que me revisen y yo les he dicho que mi esposo me pegó y no me hacen caso y, o sea, no le dan la debida canalización. Entonces cuando uno le dice a la trabajadora social: es que mi esposo me golpeó, ya nos dice: “¿sabe qué?, diríjase con esta persona”. Pero el médico, no hay ninguna respuesta (mujer de 40 años de Saltillo).

En el proceso de búsqueda de ayuda, las mujeres suelen acudir directamente al ministerio público para levantar un acta de denuncia. Los funcionarios de estas instancias suelen responder con todas las representaciones rígidas de género que contribuyen a la violencia:

Me tomaron radiografías y ya después cuando sí me vieron que estaba mal y me dijeron que a lo mejor me lo hice yo sola, que si es cierto que me lo hizo mi esposo (mujer de 44 años del Distrito Federal).

Fui al ministerio público, que vean si así, sangrando, me hacían caso [...] pero no le dan importancia, te tratan con indiferencia, con indolencia, para ellos piensan que son problemas familiares, eso ya no les importa (mujer de 35 años de Cancún).

Además, las mujeres entrevistadas experimentan desaliento por el mismo procedimiento al que deben someterse, combinado con la falta de voluntad del personal que no les evita molestias o humillaciones y que las mujeres interpretan como intentos de disuasión por parte de las instituciones. Cuando levantan un acta por lesiones, generalmente se les pide la constatación de las lesiones por parte de un médico que muchas veces no se encuentra presente. Es frecuente que se les pida regresar otro día, y solicitar otra cita. Para entonces puede ser que las lesiones ya no sean visibles, en cuyo caso se envía a la mujer de nuevo a su casa sin ninguna solución. En otras ocasiones, la misma espera hace que la propia mujer "se arrepienta" y abandone el proceso.
Algunas instituciones, incluso, alientan a la mujer a "no deshacer la familia"; se les aconseja dialogar y recomponer la situación "por el bien de los hijos":

Cuando tenía la oportunidad de salir, buscaba ayuda e incluso busqué ayuda en el DIF y me dijeron que no, pues, que ellos son para, este... que estén estables las familias, no para que las desintegren [...] Yo lo que quería era separarme, como que me quería liberar, que me protegieran de él (mujer de 37 años de Cancún).

En contraste con estas reacciones de parte de funcionarios públicos, las entrevistadas relatan experiencias muy positivas en los centros de atención a mujeres víctimas de violencia. Las mujeres entrevistadas que tienen la posibilidad de recurrir a centros de asistencia psicológica y orientación especializados, muestran signos claros de empoderamiento y la posibilidad de obtener ayuda institucional.

¿Cuándo empecé a dejar de creer que esto era normal? Cuando yo vine a Sí Mujer. Leí un folletito en donde me lo encontré por casualidad tirado, ¿verdad? Y vi la dirección. Me di cuenta de que las mujeres teníamos ciertos derechos, que no todo era lavar, planchar, sacudir y empecé a pedir ayuda, ¿verdad? (mujer de 40 años de Saltillo).

\section{Discusión}

La perspectiva subjetiva de las mujeres entrevistadas acerca de las razones a las que obedece el maltrato del cual resultan víctimas es muy importante para entender el ámbito de justificación y de atribución de responsabilidad de la violencia, como también el umbral de tolerancia vivenciado por las víctimas. Se ha observado que aquellas mujeres que explican la violencia masculina como un acto de carácter impulsivo, como expresión de frustración y sin intención de dañar, toleran más la violencia. Por el contrario, las explicaciones subjetivas que giran en torno a la violencia como castigo "merecido" por las expectativas de rol de género no cumplidas favorecen un menor grado de tolerancia a la violencia y una clara atribución de responsabilidad del maltrato a la pareja masculina. Sin embargo, este tipo de razones no conduce a la mujer a una salida pronta.

Directamente vinculadas a las razones percibidas de la violencia ejercida por el cónyuge, se pueden reconocer también las normas sociales que gobiernan la unión conyugal y que favorecen la justificación de la violencia. La reproducción de los valores y normas sociales que pautan el ejercicio tradicional de roles de 
género se pone de manifiesto también en la reacción a la violencia del entorno social y en el tipo de respuestas institucionales al problema. Con marcada frecuencia, las personas del entorno social de las mujeres golpeadas, así como los funcionarios públicos en los ámbitos de la salud y de la justicia, reaccionan con representaciones rígidas de género vinculadas al sistema de sexo-género ${ }^{20}$ dominante y no constituyen, por tanto, una fuente de apoyo social, contribuyendo además a la normalización y reproducción del problema. Este contexto social, junto con la frágil situación emocional en la que se encuentran las mujeres golpeadas, dificulta la posibilidad de encontrar una salida a la violencia.

\section{Referencias}

I. INSP/Secretaría de Salud. Encuesta Nacional sobre Violencia contra las Mujeres. ENVIM. Cuernavaca: Instituto Nacional de Salud Pública, 2003. 2. OPS/OMS. Informe mundial sobre la violencia y la salud. Pub. Científica y Técnica $\mathrm{n}^{\circ} 588$. Washington DC: OPS/OMS, 2003.

3. Heise L, Pitanguy J, Germain A.Violencia contra la mujer: la carga oculta sobre la salud. Washington D.C.: OPS, 1994.

4. Mannheim K. Strukturen des Denkens. [Estructuras del pensamiento] Frankfurt a.M.: Suhrkamp, 1980.

5. Bourdieu P. La dominación masculina. Barcelona:Anagrama, 2000. 6. Hoff $L$ A. Violence issues: an interdisciplinary curriculum guide for health professionals. Ottawa: Health Canada, 1994.
7. Glaser B, Strauss A. The discovery of grounded theory. Strategies for qualitative research. Chicago: Aldine, 1967.

8. Strauss A, Corbin J. Basics of qualitative research. Newbury Park: Sage, 1990.

9. Go V, Johnson S, Bentley M, Sivaram S, Srikrishnan A, Celentano D, et al. Crossing the threshold: engendered definitions of socially acceptable domestic violence in Chennai, India. Cult Health Sex 2003; 5: 393-408. 10. Eisikovits Z,Winstok Z, Fishman G. The first Israeli National Survey on Domestic Violence. Violence Against Women 2004 10:729-748.

II. Güezmes A, Palomino N, Ramos M. Violencia sexual y física contra las mujeres en el Perú. Estudio multicéntrico de la OMS sobre la violencia de pareja y la salud de las mujeres. Lima: OMS /Centro de la Mujer Peruana Flora Tristán /Universidad Peruana Cayetano Heredia, 2002.

12. Heise L.Violence against women: An integrated, ecological framework. Violence Against Women 1998; 4: 262-291.

13. Stets J, Straus MA.The marriage license as a hitting license: $A$ comparison of assaults in dating, cohabiting and married couples. En: Straus MA, Gelles RJ, comp. Physical violence in American families: Risk factors and adaptations to violence in 8, 145 families. New Brunswick: Transaction, 1990

14. Yllo K, Straus MA. Interpersonal violence among married and cohabiting couples. Fam Relat 198I; 30: 339-347.

15. American Medical Association. Diagnostic and treatment guidelines on domestic violence. Chicago:American Medical Association, 1994.

16. Hucpey JE. Social support: Assesing conceptual coherente. Qual Health Res 1998; 8: 304-318.

17. Bourdieu P. Le capital social: notes provisoires. Actes Rech Sci Soc 1980; 30:36.

18. Portes A. Social capital: its origins and applications in modern sociology. Ann Rev Sociol 1998; 24: I-24.

19. Rose LE, Campbell J.The role of social support and family relationships in women's responses to battering. Health Care Women Int 2000; 21: 27-40.

20. De Barbieri T. Sobre la categoría género: una introducción teóricometodológica. Isis Internacional 1992; 17: I I I-I 28. 